\title{
Ruppell's fox movement and spatial behavior are influenced by topography and human activity
}

\author{
Adi Barocas $^{1,2}$ D $\cdot$ Reuven Hefner ${ }^{3} \cdot$ Michal Ucko $^{4} \cdot$ Benny Shalmon ${ }^{3} \cdot$ Noam Leader $^{5}$. \\ Eli Geffen ${ }^{6}$
}

Received: 6 August 2020 / Revised: 6 February 2022 / Accepted: 21 February 2022 /

Published online: 4 March 2022

(c) The Author(s) 2022

\begin{abstract}
Global climatic processes have driven an expansion of arid environments, as well as the human populations that depend on these biomes. Human habitation can influence desert ecosystems directly through changes in land use and indirectly through the introduction of alien species that compete with locally adapted fauna. Native species can respond to these processes by spatial or temporal avoidance. Ruppell's fox is a desert-adapted species inhabiting flat, rocky arid areas that feeds mainly on invertebrates and rodents. We used location data and step selection functions to study the space use of a population in southern Israel. We hypothesized that Ruppell's fox movement would be influenced by topography due to preference for creeks, where insects and rodents are available, and by human infrastructure as the presence of humans, vehicles and associated animals may induce a fear effect on native species. Ruppell's fox habitat selection was seasonal. During the dry season, foxes showed preference for flatter areas and creek bottoms and avoided paved roads. During the rainy season, foxes avoided less- trafficked dirt roads. These patterns were likely a consequence of increased territoriality due to denning in the colder season and reduced availability of insects in the dry season. Avoidance of paved and dirt roads may be explained by fear of human presence and perception of risk due to the use of linear features by larger carnivores, such as wolves and red foxes. Our results suggest that current protected, remote and uninhabited areas have an elevated value for the persistence of Ruppell's fox populations. We recommend limitation of vehicle movement within current reserves and continued monitoring of population trends for this little studied carnivore.
\end{abstract}

Keywords Vulpes rueppelii · Carnivores · Resource selection · Anthropogenic activity

Communicated by Xiaoli Shen.

Adi Barocas, Reuven Hefner and Michal Ucko have contributed equally.

Adi Barocas

adibarocas@gmail.com

Extended author information available on the last page of the article 


\section{Introduction}

Increasing levels of human activity influence the spatial behavior of animal populations throughout the planet (Tucker et al. 2018). in arid mountainous regions, the effects of human infrastructure may be intensified because people concentrate their activities along valley bottoms, which also provide the best habitat for many species (Suraci et al. 2019). While human activities may cause direct mortality, habitat degradation, and habitat loss, they also may inhibit animal movements between prime foraging areas. To mitigate the impacts of habitat loss and fragmentation, it is necessary to determine the spatial response of animals to different types of anthropogenic development such as roads, trails, fields, and towns (Wang et al. 2015).

The combination of roads, trails, and other human developments may have negative influence on carnivores because these species occur in low densities and occupy large home ranges that typically encompass multiple anthropogenic obstacles (Crooks 2002). Because the density of human infrastructure within their home range is variable (Riley et al. 2003), carnivores may differ in their responses to human development. Roads typically cause habitat loss and fragmentation but can also be used to facilitate animal movement. Their use for hunting, for example, may vary according to vehicle traffic and to adjacent landscape features and topography (Whittington et al. 2005; Houle et al. 2010). Linear features may provide protection for smaller carnivores and prey species from top predators (Berger 2007) or increase their predation risk (Whittington et al. 2011; Prokopenko et al. 2017).

Arid environments have expanded globally in recent decades (Maestre et al. 2012). Both human activities and the expansion of commensal species that accompany them have driven ecological changes in desert landscapes, including declines in native species and trophic disruptions (Schlesinger et al. 1990; D'Odorico et al. 2013). Despite the increase in human populations that depend on arid ecosystems, these areas remain relatively understudied for logistic and cultural reasons (Pyšek et al. 2008; Brito et al. 2009). Specifically, knowledge of the distribution and spatial behavior of mammalian carnivores in desert areas is deficient (Brito et al. 2009).

Ruppell's fox (Vulpes rueppellii) is a small carnivore, adapted to arid environments. A limited number of robust studies of its ecology and behavior suggest that it has an omnivorous diet, based mostly on insects and rodents, and lives in pair-based territories, of circa $30 \mathrm{~km}^{2}$. Ruppell's fox range encompasses several remote and protected areas. Its habitat preferences, however, are not well understood (Lindsay and Macdonald 1986; Kowalski 1988; Murdoch et al. 2007; Mallon et al. 2015). In Northern Africa, distribution models suggest a partitioning of habitat preferences between Ruppell's fox and the red fox (Vulpes vulpes). In this system, Ruppell's fox has high habitat suitability throughout the Sahara desert, but is mostly found in areas with low precipitation and at short distances from stony desert (Brito et al. 2009). In Israel, Ruppell's fox is distributed throughout wide washes and valleys in the arid South. Its range is partially covered by protected areas and military training zones, and spans a number of small settlements (Mendelssohn and Yom-Tov 1999).

We used GPS location data to study the movement and spatial behavior of a wild population of Ruppell's fox that inhabits the Negev desert. We hypothesized that Ruppell's foxes would show avoidance of human settlements and linear features such as paved and dirt roads resulting from human activity, perceived as high-risk areas because of the possibility of encountering vehicles and the presence of larger predators. We additionally predicted that Ruppell's foxes would favor areas, such as valley bottoms and creek beds, where 
they would be able to forage minimizing exposure to larger carnivores, and where the probability of finding invertebrates and rodents would be higher. We predicted that this preference would increase during the dry season, when insects, a key part of Ruppell's fox diet, display higher nocturnal activity.

\section{Methods}

\section{Research area}

The study was conducted in the extremely arid Negev desert, where mean daily temperatures range between $12.5{ }^{\circ} \mathrm{C}$ in January to $30.5{ }^{\circ} \mathrm{C}$ in July and mean annual precipitation is $95.8 \mathrm{~mm}$ (http://www.ims.gov.il). The study area extended from the settlement of Paran $\left(30.554^{\circ} \mathrm{N}, 35.189^{\circ} \mathrm{E}\right)$ in the North to Grofit $\left(29.936^{\circ} \mathrm{N}, 35.067^{\circ} \mathrm{E}\right)$ in the South, mostly within the borders of Israel, with a limited extent of Egypt included (Fig. 1). The area, a part of the Rift Valley, presents wide, savanna-like washes, characterized by shrubs and acacia (Vachellia sp.) trees. The area contains seven settlements totaling 1815 people, whose livelihoods are mostly from agriculture, tourism and limited dairy production, adjacent to the main road to Eilat, as well as a number of military bases (Sagie et al. 2013).

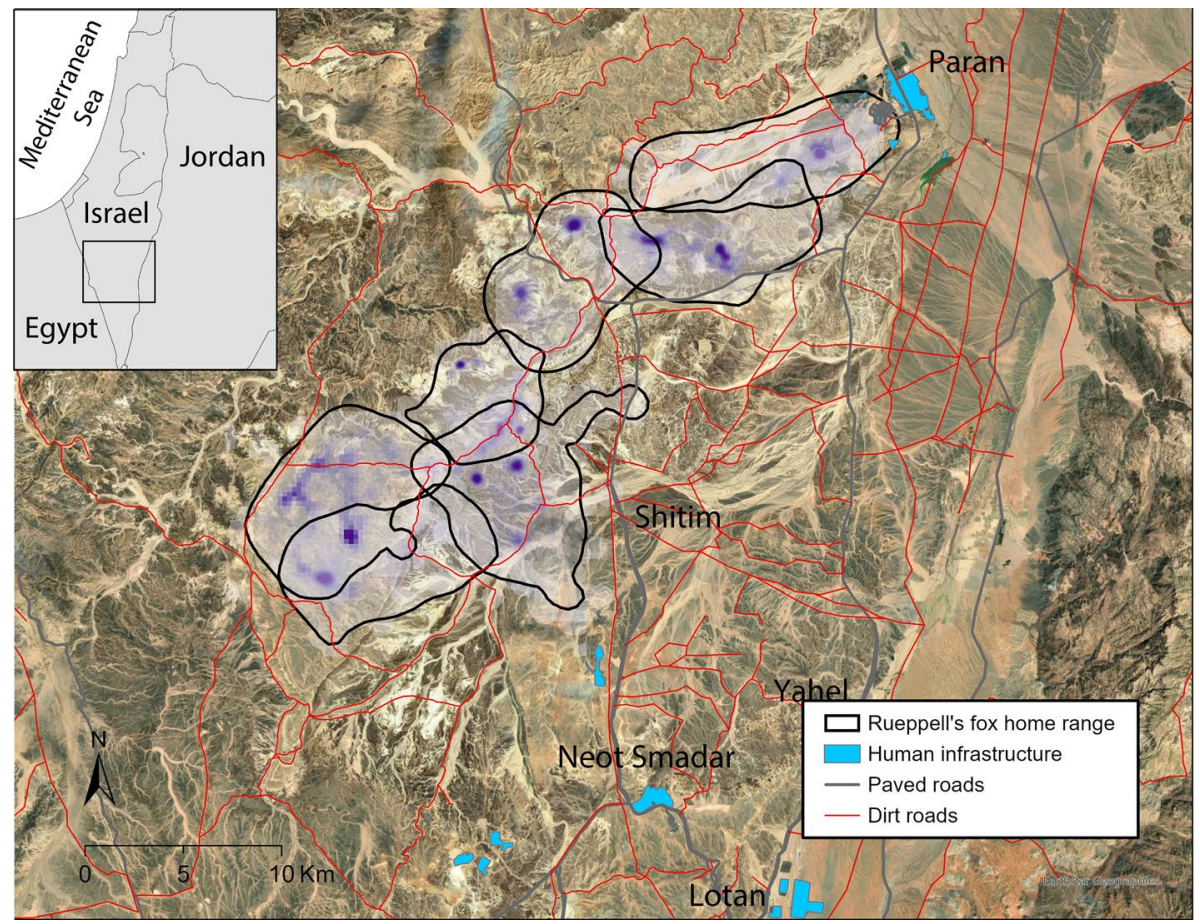

Fig. 1 Map of Ruppell's fox research area in southern Israel, including portions of Egypt and Jordan. Contours represent individual 95\% Kernel home ranges, shown for 7 out of 24 individual Ruppell's foxes for clarity. Blue-colored polygons denote areas of human activity. The white to purple gradient shows kernel density for each territory based on GPS locations. Fox location data was collected in southern Israel between 2016 and 2019 
Parts of the research area are protected by the Nehalim Gedolim and Eshush reserves (Fig. $\mathrm{S} 1$ ). Human-associated carnivore species are the striped hyena (Hyaena hyaena), the Arabian wolf (Canis lupus arabs), the red fox and golden jackal (Canis aureus; Cohen et al. 2013; Lewin et al. 2021).

\section{Animal capture}

We captured Ruppell's foxes using cage traps (Tomahawk Live Trap Company, Tomahawk, WI, USA), baited with dead chicks, in three different wide wash areas of the Negev Desert. In each trap session we deployed 10-20 traps at intervals of 0.5-2 km, along a transect of about $14 \mathrm{~km}$. We left traps open during the night and examined them each morning for 5-10 consecutive days. Captured individuals were sexed, weighted, measured, eartagged and fit with ATS (Advanced Telemetry Systems Inc. ATS, Isanti, Minnesota, USA) or Lotek (Lotek Engineering Systems, Newmarket, Ontario, CA) GPS collars. Collars were programmed to obtain hourly fixes during the evening and night (6 pm-6 am). Animals fitted with GPS collars were subsequently recaptured for data recovery. In addition, we performed opportunistic tracking sessions with custom-made antennas to locate tagged animals and download data. All animal handling procedures complied with the permits from the Israel Nature and Parks Authority (INPA; 2017/41654/, 2018/41975, 2019/42380).

\section{Habitat covariates and home range estimation}

To calculate fox home ranges, we used two methods: minimum convex polygon (MCP), based on the bounding feature of the most peripheral points, and Kernel Utilization Density (KUD), representing the density probability of use by individuals in each pixel (Seaman and Powell 1996). For each of these methods, we delineated 50\%, 95\% and 99\% contours. We present home range areas for $95 \%$ contours to account for the variability of habitat available to each individual, while mitigating the effects of single long-distance movements and outlying points (Kie et al. 2010).

To assess the influence of human activity on fox space use and movement, we developed GIS layers quantifying distance from anthropogenic features, and distance from paved and dirt roads. We did not separately quantify distance from residential areas, agriculture and military bases because these distances are highly correlated in the research area (Barocas et al. 2018). We obtained human infrastructure and road data from the INPA (Table 1). To quantify elevational and topographical variation within fox home ranges, we developed layers depicting elevation, slope, aspect and topographic position (Table 1). All layers had pixel resolution of $30 \mathrm{~m}$.

\section{Step selection functions}

To investigate the factors that influence the movement of Ruppell's foxes at the home range and patch levels, and whether movement varied among periods of activity, we considered the straight-line distances travelled by foxes between hourly fixes as steps. We used recorded sunset and sunrise hours to determine times of activity and divided Ruppell's fox fix relocation data into a twilight, a night, and a morning dataset. Because the majority of points were recorded during night time, to examine possible effects of seasonality, we divided the night time dataset to two seasons based on rain and temperature records: a warmer dry season, 


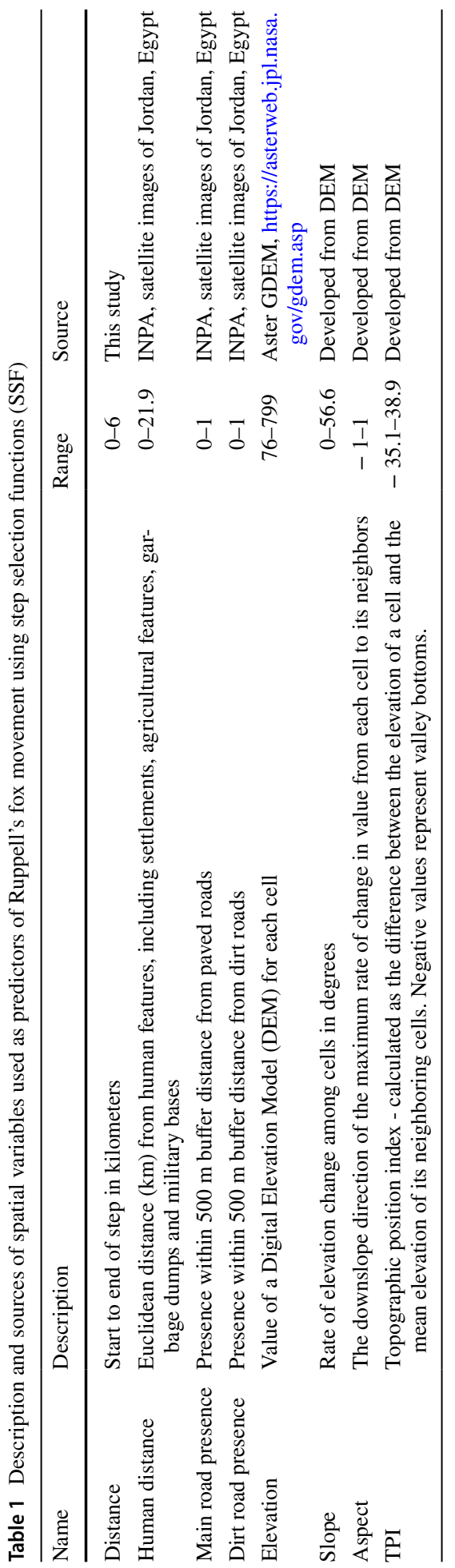


lasting between May and September and a colder 'rainy' season, in which there was some probability of rain in the area, between October and April (www.timeanddate.com).

For each period and season, we built a separate step selection function (SSF; Fortin et al. 2005). We were interested in both true movement and behavior. Thus, we ran SSFs both including and excluding steps shorter than $50 \mathrm{~m}$, accounting for telemetry error and periods of limited movement. We used a matched case-control design, generating 20 available steps, each starting from the same point as the observed step. We based the length and angle of available steps on the observed distribution of step lengths and turning angles of the individual's matching sampled observed steps. For each observed or available step, we extracted habitat covariates at the end point of each step (Thurfjell et al. 2014). After testing all pairs of variables for collinearity, we fitted five models. The null model included only step length (coded as the Distance covariate); The Human Activity model included distance from human infrastructure and presence/absence within $500 \mathrm{~m}$ of paved and dirt roads; The Topography model included elevation, slope, aspect, and topographic position; The Full models combined both human activity and topography-related variables (Table 2). Models were parametrized using conditional logistic regression, with each stratum identified as a used point and its 20 paired available points. We calculated robust SE and 95\% CI of parameters using generalized estimating equations (GEE; Craiu et al. 2008). Because data were continuous for all foxes and rarely spanned above 150 days (Table S1), clusters in the GEE were defined according to individual. We compared the fit of the four competing models with the null model using quasilikelihood information criteria (QIC). QIC is based on similar principles as the Akaike information criterion (AIC), maximizing model likelihood but penalizing for a large quantity of estimable parameters (Burnham and Anderson 2002). QIC is adapted for correlated response data, being based on quasi-likelihood estimation (Pan 2001). For each time period examined, we present the selection coefficient, standard errors and P-values as suggested by the output of the best-supported model. In one case, when $\Delta \mathrm{QIC}<10$, indicating some support for competing models, we also present model-averaged coefficients (Burnham et al. 2011).

We validated the robustness of SSFs using a five-fold cross-validation repeated 100 times, following the framework developed by Fortin et al. (2009) for SSFs. In brief, this method is intended for a case-control design, in which the SSF is built using a randomly selected $80 \%$ of the data and it is used to estimate predicted values of the withheld $20 \%$ of data. Using the predicted values, the observed location of each stratum is ranked against its associated available steps (from 1 to 11, i.e., the observed step and its paired 10 available steps). Ranks of observed steps are tallied into 11 bins, and a Spearman rank correlation $\left(r_{s}\right)$ is employed to test whether the highest ranked bins include the largest number of observed steps. We also assessed the mean range of expected $r_{s}$ (i.e., a random choice of points) based on the above methods, but instead of ranking the observed step against the 11 possible, we randomly selected one of the ten available steps for each stratum (Fortin et al. 2009). All analyses were performed in the R computational environment (version 3.6.5; R Core Team 2019).

\section{Results}

We captured 24 Ruppell's foxes, 14 females and 10 males. We obtained 35,638 successful fixes $(84.7 \%$ success rate), for a mean $\pm S E$ of $1,486.6 \pm 184.6$ fixes per individual and a tracking period of mean \pm SE $129.4 \pm 16.5$ days. 95\% kernel UD estimates of fox home ranges averaged $66.5 \mathrm{~km}^{2},( \pm \mathrm{SE}=12.2)$, slightly higher compared to $95 \%$ minimum convex polygons estimates (mean \pm SE $63.3 \pm 13.6 \mathrm{~km}^{2}$; Table S1). Two individuals 


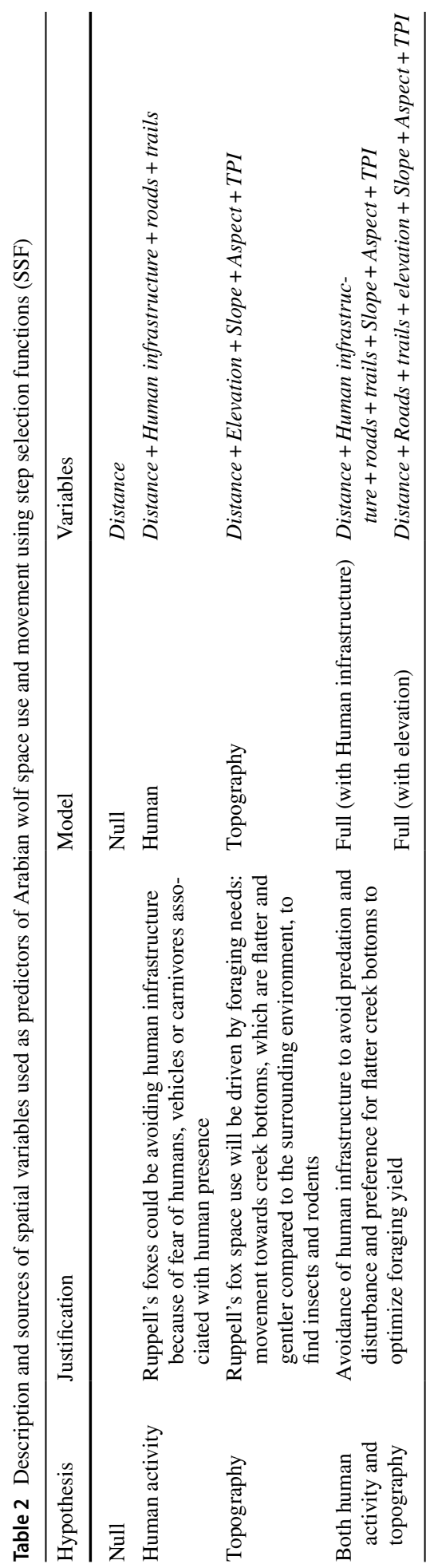


demonstrated long movements, and their home range areas were disproportionally large, possibly indicating dispersal-related behavior (255.9 and $244.9 \mathrm{~km}^{2}$ ). Three fox home ranges included the small settlement of Paran and one included the Shitim settlement (Fig. 1, Fig. S1). Home ranges also spanned dirt roads, military bases, garbage dumps and agricultural areas (Fig. S1).

\section{Step selection functions}

For Ruppell's fox movement SSFs, elevation and distance from human infrastructure were highly correlated (Spearman $r>0.7$ ) in all datasets. Thus, to avoid collinearity, we built models including each one of these and all additional covariates. We obtained 4205 steps for the twilight period, 9379 during night and 508 in the dawn and morning. The night dataset included 4412 steps in the dry season and 5061 in the rainy season. During twilight, the Topography model was best supported $\left(\Delta \mathrm{QIC}_{\text {all others }} \geq=28.5\right.$; Table $\mathrm{S} 2$ ), outperforming full models and models with variables reflecting human activity. Model output suggested that Ruppell's foxes moved towards gentler slopes and lower elevations (Tables S3, S4). The night dataset results differed by season. During the dry season, Ruppell's foxes avoided paved roads and moved towards higher aspect values and valley bottoms (Tables S5, S6, S7, Fig. 2). During the rainy season, the best supported models included all variables except distance from human infrastructure $\left(\Delta \mathrm{QIC}_{\text {full vs. null }}=104.6\right.$; Table $\left.\mathrm{S} 9\right)$. When steps smaller than $50 \mathrm{~m}$ were included, Ruppell's foxes showed avoidance of dirt roads, and moved towards gentler slopes, lower aspects, and valley bottoms (Tables S10, S11; Fig. 3). During dawn and morning, the model including all variables except distance from human infrastructure, performed best $\left(\Delta \mathrm{QIC}_{\text {elevation vs. null }}=88.7\right.$; Table S12). During mornings, Ruppell's foxes also showed preference for steeper slopes $(\beta=0.05 \pm 0.005$; $\mathrm{P}<0.001$; Tables S13, S14).

All models outperformed null models with distance as the only independent factor (Tables S2, S5, S8, S11). During the majority of periods, results excluding steps shorter than $50 \mathrm{~m}$ did not differ from models including all steps. During twilight, the excluded model suggested that Ruppell's foxes favored lower elevations and reduced slopes (Table S4). During night, in the dry season, all coefficients were similar for both datasets, except the distance covariate (Tables S7, S8). In the rain season, removal of shorter steps $(n=603 ; 12 \%$ of steps) increased the significance of some coefficients, indicating

(a)

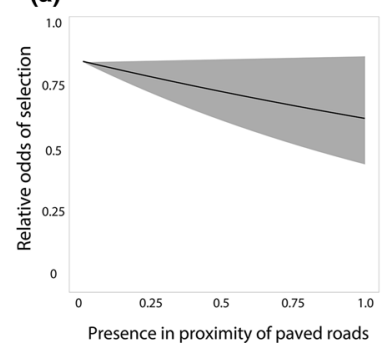

(b)

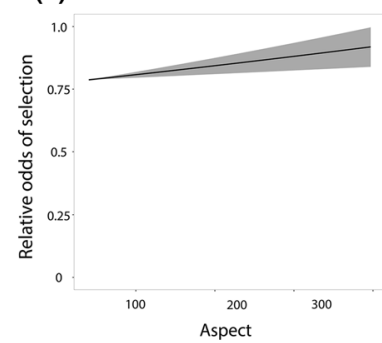

(c)

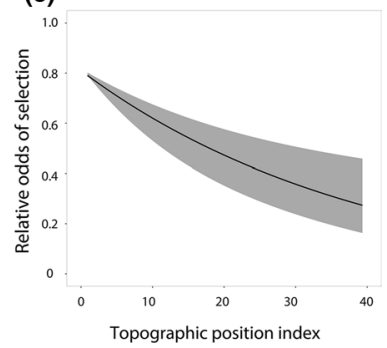

Fig. 2 Relative odds (exponent [exp] of selection coefficient) of selection as a function of distinct covariates during night hours fit to Ruppell's fox movement data in the dry season. Full lines represent predicted relationships using mean selection coefficients, with shaded areas representing the $95 \%$ CI of the prediction. SSF was fit to GPS locations from 24 Ruppell's foxes, collected in 2016-2019 in southern Israel 
(a)

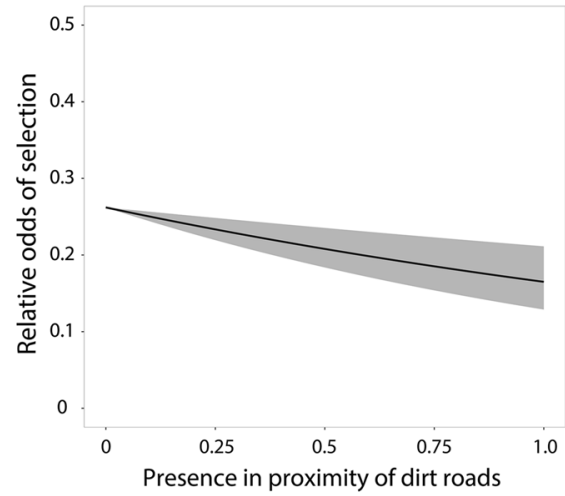

(c)

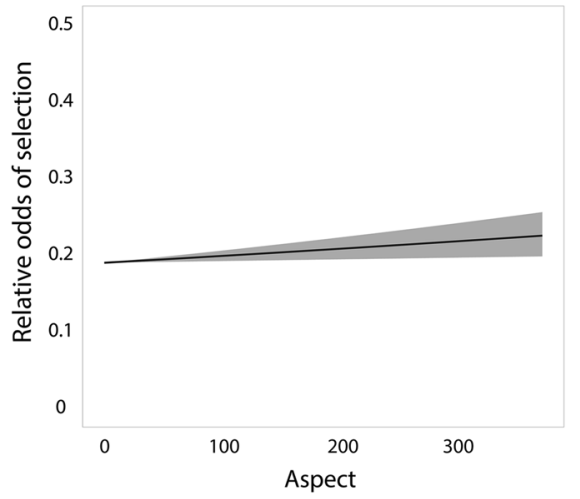

Wet season

(b)

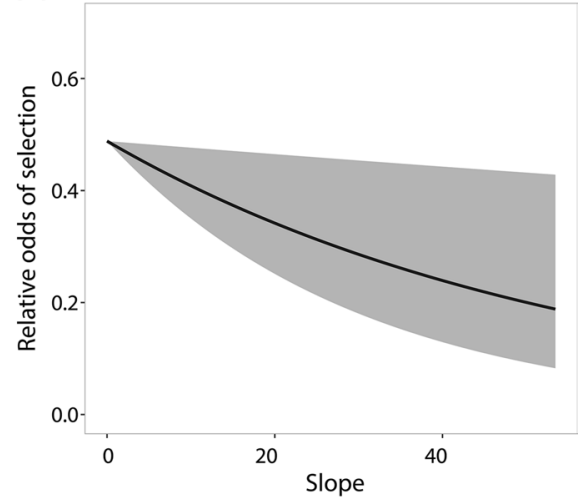

(d)

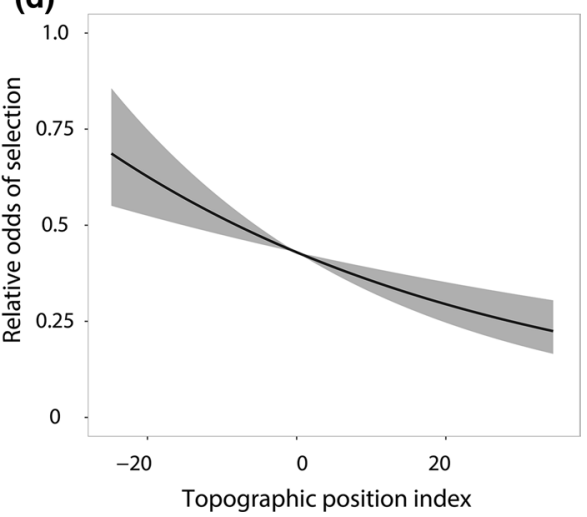

Fig. 3 Relative odds (exponent [exp] of selection coefficient) of selection as a function of distinct covariates during night hours fit to Ruppell's fox movement data in the rainy season. Full lines represent predicted relationships using mean selection coefficients, with shaded areas representing the $95 \%$ CI of the prediction. SSF was fit to GPS locations from 24 Ruppell's foxes, collected in 2016-2019 in southern Israel

preference for lower elevations, lower slopes, higher aspect values and valley bottoms (Tables S10, S11; Fig. 3). Cross-validation results indicated good fit of best-supported models for each period (twilight $r_{s}=0.88 \pm 0.04$, random $=0.03 \pm 0.24$; dry season night $r_{s}=0.82 \pm 0.06$, random $=0.01 \pm 0.21$; rainy season night $r_{s}=0.82 \pm 0.06$, ran$\operatorname{dom}=0.01 \pm 0.23$; morning $r_{s=} 0.64 \pm 0.11$, random $=-0.01 \pm 0.26$ ).

\section{Discussion}

Ruppell's fox preference for landscape features varied according to time of day and season. Movement data showed that, during the dry season, topography and linear features had a strong influence on fox movements, with significant avoidance of paved roads within their home ranges. During the rainy season, Ruppell's foxes showed avoidance of dirt roads and, during movement, favored lower slopes and flatter areas, representing valley bottoms. Thus, our data supported both the topography and the human 
activity hypotheses. In addition, an analysis of 50 diet samples suggested that Ruppell's foxes mostly feed on invertebrates and rodents. Fox diets contained low proportions of human-derived items (i.e. garbage), suggesting little dependence on human subsidies (Text S1, Fig. S2).

Research suggests that fear inspired by humans is stronger than the effect of large or introduced carnivores (Clinchy et al. 2016). Ruppell's fox avoidance of human infrastructure varied among seasons and time periods. Twilight patterns probably reflected emergence from dens to begin activity and movement towards foraging areas in creek bottoms. During the night, the main foraging period for the species (Lindsay and Macdonald 1986), individuals avoided dirt and paved roads and favored locations with gentle topography, characterized by reduced slopes and valley bottoms, especially in the dry season. The preference for higher slopes during morning hours probably reflected movement towards dens, located in heightened areas (R. Hefner, unpublished data). Depending on season, at night Ruppell's foxes avoided either the proximity of dirt or paved roads used by humans. In addition, they were rarely found within or near human infrastructure such as garbage dumps and fields. This may be explained by the increased occurrence of commensal carnivores in the vicinity of human infrastructure (unpublished data) and the use of trails and roads by the nocturnal Arabian wolf, this area's top carnivore (Barocas et al. 2018). Such areas may be perceived as risky by Ruppell's foxes. Similarly, moose (Alces alces) avoided anthropogenic linear features used preferentially for movement by their predators, suggesting increased perceived risk (Dickie et al. 2020). Taken together, our findings suggest that during the dry season, the availability of lower altitude, flat valley bottoms, likely containing vegetation patches with invertebrates and rodents, is the most significant driver of Ruppell's fox movement. Thus, during the warm months foraging decisions are primarily driven by food patches and risk presented by paved and dirt roads, and only secondarily by the proximity of human infrastructure.

Ruppell's foxes in the Arabian peninsula and the Sahara are commonly found in a variety of rocky, gravelly and sandy desert habitats with little vegetation cover and reduced rainfall (Lenain 2000; Brito et al. 2009). Our findings of preference for flatter, more valley-like topography are in line with such evidence. Home range sizes (mean $66.5 \mathrm{~km}^{2}$ ) were twice as large as those found in previous studies, suggesting that Negev foxes may be more mobile compared to other populations. Alternatively, the relative detail and length of our study may provide more accurate home range estimates compared to previous findings (Lindsay and Macdonald 1986; Murdoch et al. 2007). Ruppell's fox diet samples provide limited inference. However, they do suggest preference for invertebrates and small mammals and a rarity of human-derived items (Fig. S2). Thus, our results do not support an increased presence of Ruppell's foxes near human settlements or higher densities of the species' populations in human vicinity, as suggested by some studies (Mallon et al. 2015). The findings of seasonal movement patterns can be explained by changes in resource availability and breeding-related behavior. During colder months, lower temperatures may limit the availability of invertebrates during night hours, altering Ruppell's fox spatial behavior and potentially increasing time spent foraging. In the Negev, the rainy season also coincides with periods when Ruppell's foxes raise their cubs (February-March; R. Hefner, unpublished data), during which they may be more territorial and avoid potentially riskier areas. In addition, despite the lack of support for avoidance of or preference for human infrastructure, during colder months, settlements, where Ruppell's foxes have increased probability of encountering wolves (Barocas et al. 2018) and red foxes (unpublished data), may become less risky because these two larger carnivores may favor areas with temporary local vegetation blooms, where the probability of finding animal prey is higher. 
Ruppell's fox home ranges were mostly covered by the large Nehalim Gedolim and Eshush protected areas. However, our results suggest several movements outside these areas, within military training areas and in the proximity of paved roads (Fig. S1). Given the observed avoidance of dirt roads, found within all Ruppell's fox home ranges (Fig. 1, Fig. S1), to mitigate anthropogenic effects on Ruppell's fox habitat, efforts should be made to limit the movement of vehicles visiting these areas. Vehicle travel should be restricted to dirt roads and designated camping and parking sites. We conclude that, in southern Israel, undisturbed and protected areas have an elevated importance because of their ability to ensure the stability of local fox populations in the face of expanding human activity.

In the Negev, as well as throughout its range, Ruppell's fox appears to benefit from the isolation and aridity of the ecosystems it inhabits, including wide creeks and microhabitats that contain vegetation patches crucial for the species' survival (Brito et al. 2009). However, given the significant year-to-year fluctuations in the number of captured individuals (E. Geffen, unpublished data), local and regional population trends are still not well understood and demographic monitoring should be continued in order to secure the species' conservation (Cuzin and Lenain 2004; Mallon et al. 2015). As human populations grow and arid areas occupied by settlements and agriculture expand, desert carnivores such as Ruppell's fox may be subject to competition and range reduction. Because several local small carnivore species in arid environments are negatively influenced by human-associated carnivores (Ritchie and Johnson 2009), examining the ecological and behavioral interactions between Ruppell's fox, the red fox, the golden jackal and the Arabian wolf could constitute a promising research direction and contribute to the species' conservation.

Supplementary Information The online version contains supplementary material available at https://doi. org/10.1007/s10531-022-02395-9.

Acknowledgements We thank the many field volunteers, especially Roy Talbi and Shachar Parker and Israel Nature and Parks Authority rangers especially Moran Bakish for their valuable help. Ofer Steinitz from INPA provided data on carnivore observations. Funding and logistic support were provided by the INPA. We are grateful to Naomi Paz for linguistic edits.

Funding Funding and logistic support for the research were provided by the Israel Nature and Parks Authority.

Data availability Ruppell's fox location data will be deposited in a digital repository upon acceptance for publication (e.g. Movebank, www.movebank.org).

Code availability Not applicable.

\section{Declarations}

Conflict of interest Not applicable.

Ethical approval All animal handling procedures complied with the permits of the Israel Nature and Parks Authority (INPA; 2017/41654/, 2018/41975, 2019/42380).

Consent to participate Not applicable.

Consent for publication Not applicable. 
Open Access This article is licensed under a Creative Commons Attribution 4.0 International License, which permits use, sharing, adaptation, distribution and reproduction in any medium or format, as long as you give appropriate credit to the original author(s) and the source, provide a link to the Creative Commons licence, and indicate if changes were made. The images or other third party material in this article are included in the article's Creative Commons licence, unless indicated otherwise in a credit line to the material. If material is not included in the article's Creative Commons licence and your intended use is not permitted by statutory regulation or exceeds the permitted use, you will need to obtain permission directly from the copyright holder. To view a copy of this licence, visit http://creativecommons.org/licenses/by/4.0/.

\section{References}

Barocas A, Hefner R, Ucko M et al (2018) Behavioral adaptations of a large carnivore to human activity in an extremely arid landscape. Anim Conserv 21:433-443

Berger J (2007) Fear, human shields and the redistribution of prey and predators in protected areas. Biol Lett 3:620-623. https://doi.org/10.1098/rsbl.2007.0415

Brito JC, Acosta AL, Álvares F, Cuzin F (2009) Biogeography and conservation of taxa from remote regions: an application of ecological-niche based models and GIS to North-African Canids. Biol Conserv 142:3020-3029. https://doi.org/10.1016/j.biocon.2009.08.001

Burnham K, Anderson A (2002) Model selection and multimodel inference: a practical informationtheoretic approach. Springer, New York

Burnham KP, Anderson DR, Huyvaert KP (2011) AIC model selection and multimodel inference in behavioral ecology: some background, observations, and comparisons. Behav Ecol Sociobiol $65: 23-35$

Clinchy M, Zanette LY, Roberts D et al (2016) Fear of the human "super predator" far exceeds the fear of large carnivores in a model mesocarnivore. Behav Ecol 27:1826-1832. https://doi.org/10.1093/beheco/ arw 117

Cohen O, Barocas A, Geffen E (2013) Conflicting management policies for the Arabian wolf Canis lupus arabs in the Negev Desert: is this justified? Oryx 47:228-236. https://doi.org/10.1017/S003060531 1001797

Craiu RV, Duchesne T, Fortin D (2008) Inference methods for the conditional logistic regression model with longitudinal data. Biometrical J 50:97-109. https://doi.org/10.1002/bimj.200610379

Crooks KR (2002) Relative sensitivities of mammalian carnivores to habitat fragmentation. Conserv Biol 16:488-502

Cuzin F, Lenain DM (2004) Rüppell's fox (Vulpes rueppellii). In: Sillero-Zubiri C, Hoffmann M, MacDonald DW (eds) Canids: Foxes, Wolves, Jackals, and Dogs. Status survey and conservation action plan. IUCN/SSC Canid Specialist Group, Gland, Switzerland, pp 201-204

Dickie M, McNay SR, Sutherland GD et al (2020) Corridors or risk? Movement along, and use of, linear features varies predictably among large mammal predator and prey species. J Anim Ecol 89:623-634

D'Odorico P, Bhattachan A, Davis KF et al (2013) Global desertification: drivers and feedbacks. Adv Water Resour 51:326-344

Fortin D, Beyer HL, Boyce MS et al (2005) Wolves influence elk movements: behavior shapes a trophic cascade in Yellowstone National Park. Ecology 86:1320-1330

Fortin D, Fortin ME, Beyer HL et al (2009) Group-size-mediated habitat selection and group fusion-fission dynamics of bison under predation risk. Ecology 90:2480-2490. https://doi.org/10.1890/08-0345.1

Houle M, Fortin D, Dussault C et al (2010) Cumulative effects of forestry on habitat use by gray wolf (Canis lupus) in the boreal forest. Landsc Ecol 25:419-433. https://doi.org/10.1007/s10980-009-9420-2

Kie JG, Matthiopoulos J, Fieberg J et al (2010) The home-range concept: are traditional estimators still relevant with modern telemetry technology? Philos Trans R Soc B. https://doi.org/10.1098/rstb.2010.0093

Kowalski K (1988) The food of the sand fox Vulpes rueppelli Schinz, 1825 in the Egyptian Sahara. Folia Biol 36:1-2

Lenain DM (2000) Fox populations of a protected area in Saudi Arabia

Lewin A, Erinjery JJ, de Waroux Y, le P et al (2021) Land-use differences modify predator-prey interactions and Acacia vegetation in a hyperarid ecosystem. J Arid Environ 192:104547

Lindsay IM, Macdonald DW (1986) Behaviour and ecology of the Ruppell's fox, Vulpes ruppelli, in Oman. Mammalia 50:461-474

Maestre FT, Salguero-Gomez R, Quero JL (2012) It is getting hotter in here: determining and projecting the impacts of global environmental change on drylands. Philos Trans R Soc B 367:3062-3075 
Mallon D, Murdoch JD, Wacher T (2015) Vulpes rueppellii. The IUCN red list threat species e.T23053A46197483. https://doi.org/10.2305/IUCN.UK.2015

Mendelssohn H, Yom-Tov Y (1999) A report of birds and mammals which have increased their distribution and abundance in Israel due to human activity. Isr J Zool 45:35-47

Murdoch JD, Drew C, Llanes IB, Tourenq C (2007) Rüppell's foxes in Al Dhafra, United Arab Emirates. Canid News 10(1)

Pan W (2001) Akaike's information criterion in generalized estimating equations. Biometrics 57:120-125

Prokopenko CM, Boyce MS, Avgar T (2017) Characterizing wildlife behavioural responses to roads using integrated step selection analysis. J Appl Ecol 54:470-479

Pyšek P, Richardson DM, Pergl J et al (2008) Geographical and taxonomic biases in invasion ecology. Trends Ecol Evol 23:237-244

R Core Team R (2019) R: a language and. environment for statistical computing. Vienna, Austria

Riley SPD, Sauvajot RM, Fuller TK et al (2003) Effects of urbanization and habitat fragmentation on bobcats and coyotes in southern California. Conserv Biol 17:566-576

Ritchie EG, Johnson CN (2009) Predator interactions, mesopredator release and biodiversity conservation. Ecol Lett 12:982-998. https://doi.org/10.1111/j.1461-0248.2009.01347.x

Sagie H, Morris A, Rofè Y et al (2013) Cross-cultural perceptions of ecosystem services: a social inquiry on both sides of the Israeli-Jordanian border of the Southern Arava Valley Desert. J Arid Environ 97:38-48

Schlesinger WH, Reynolds JF, Cunningham GL et al (1990) Biological feedbacks in global desertification. Science 247:1043-1048

Seaman DE, Powell RA (1996) An evaluation of the accuracy of kernel density estimators for home range analysis. Ecology 77:2075-2085

Suraci JP, Clinchy M, Zanette LY, Wilmers CC (2019) Fear of humans as apex predators has landscapescale impacts from mountain lions to mice. Ecol Lett 22:1578-1586

Thurfjell H, Ciuti S, Boyce MS (2014) Applications of step-selection functions in ecology and conservation. Mov Ecol 2:4. https://doi.org/10.1186/2051-3933-2-4

Tucker MA, Böhning-Gaese K, Fagan WF et al (2018) Moving in the anthropocene: global reductions in terrestrial mammalian movements. Science 359:466-469. https://doi.org/10.1126/science.aam9712

Wang Y, Allen ML, Wilmers CC (2015) Mesopredator spatial and temporal responses to large predators and human development in the Santa Cruz Mountains of California. Biol Conserv 190:23-33

Whittington J, Clair CCS, Mercer G (2005) Spatial responses of wolves to roads and trails in mountain valleys. Ecol Appl 15:543-553. https://doi.org/10.1890/03-5317

Whittington J, Hebblewhite M, Decesare NJ et al (2011) Caribou encounters with wolves increase near roads and trails: a time-to-event approach. J Appl Ecol 48:1535-1542. https://doi.org/10.1111/j.13652664.2011.02043.x

Publisher's Note Springer Nature remains neutral with regard to jurisdictional claims in published maps and institutional affiliations.

\section{Authors and Affiliations}

\section{Adi Barocas ${ }^{1,2}$. Reuven Hefner ${ }^{3} \cdot$ Michal Ucko $^{4} \cdot$ Benny Shalmon ${ }^{3} \cdot$ Noam Leader $^{5}$. Eli Geffen ${ }^{6}$}

1 San Diego Zoo Wildlife Alliance, 15600 San Pasqual Valley Road, 92027 Escondido, CA, USA

2 Wildlife Conservation Research Unit, Department of Zoology, University of Oxford, Tubney, Abingdon OX13 5QL, UK

3 Israel Nature and Parks Authority, Southern District, 84215 Beer Sheva, Israel

4 Israel Oceanographic and Limnological Research, National Center for Mariculture, PO Box 1212, 88112 Eilat, Israel

5 Ecology Department, Science Division, Israel Nature and Parks Authority, 95463 Jerusalem, Israel

6 School of Zoology, Tel Aviv University, 69978 Tel Aviv, Israel 